\title{
Comparison between preoperative two-dimensional shear wave elastography and indocyanine green clearance test for prediction of post-hepatectomy liver failure
}

\author{
Tingting Qiu ${ }^{1}$, Rong Fu ${ }^{1}$, Wenwu Ling ${ }^{1}$, Jiawu Li $^{1}$, Jiulin Song ${ }^{2}$, Zhenru Wu ${ }^{3}$, Yujun Shi ${ }^{3}$, Yuqing Zhou ${ }^{1}$, \\ Yan Luo 1 \\ ${ }^{1}$ Department of Medical Ultrasound, West China Hospital of Sichuan University, Chengdu, China; ${ }^{2}$ Department of Hepatology, West China \\ Hospital of Sichuan University, Chengdu, China; ${ }^{3}$ Laboratory of Pathology, Key Laboratory of Transplant Engineering and Immunology, NHC, \\ West China Hospital, Sichuan University, Chengdu, China
}

Correspondence to: Yan Luo, MD. Department of Medical Ultrasound, West China Hospital of Sichuan University, Chengdu 610041, China. Email: luoyanddoc@163.com.

Background: Post-hepatectomy liver failure (PHLF) is one of the most serious complications and major causes of liver resection mortality. The purpose of this study is to investigate and compare the performance of preoperative two-dimensional shear wave elastography (2D-SWE) and the indocyanine green (ICG) clearance test for the prediction of PHLF.

Methods: A total of 172 consecutive patients who were undergoing major liver resection were prospectively identified. Patients were evaluated by preoperative 2D-SWE and ICG clearance test. According to the International Study Group of Liver Surgery (ISGLS) recommendations, No PHLF, PHLF A, PHLF B, and PHLF C group classifications were defined. The differences in liver stiffness value (LSV) and ICG retention rate at 15 minutes (ICGR15) among the different PHLF classifications were investigated. The performance of LSV and ICGR15 for diagnosing different classifications of PHLF was compared.

Results: PHLF occurred in 43 (25.0\%) patients, and 24 (14.0\%) patients were grade A, 14 (8.1\%) were grade B, and 5 (2.9\%) were grade C. Both LSV and ICGR15 of the PHLF C group were significantly higher than those of the No PHLF group ( $\mathrm{P}=0.025, \mathrm{P}=0.001$, respectively). According to univariate and multivariate logistic regression analysis, LSV and ICGR15 were significantly related to PHLF $(\mathrm{P}=0.051, \mathrm{P}=0.084$, respectively). For diagnosis of $\geq$ PHLF A, $\geq$ PHLF B, and $\geq$ PHLF C, the areas under the receiver operating characteristic curve (AUCs) for 2D-SWE were 0.624 [95\% confidence interval (CI): 0.536-0.712, P=0.015], 0.699 (95\% CI: 0.576-0.821, P=0.005), and 0.831 (95\% CI: 0.737-0.925, P=0.01), respectively. The AUCs of the ICG clearance test were 0.631 (95\% CI: 0.542-0.721, P=0.01), 0.570 (95\% CI: 0.436-0.704, P=0.32), and 0.717 (95\% CI: 0.515-0.920, P=0.098), respectively. The AUC of LSV for the diagnosis of $\geq$ PHLF A was comparable to that of ICGR15 ( $\mathrm{P}=0.17)$. The AUCs of LSV were significantly higher than those of ICGR15 for the diagnosis of $\geq$ PHLF B $(\mathrm{P}=0.002)$ and $\mathrm{C}(\mathrm{P}=0.038)$.

Conclusions: 2D-SWE demonstrates the potential to aid in the prediction of the severity of PHLF. Our findings also suggest that the performance of 2D-SWE is better than the ICG clearance test.

Keywords: Shear wave elastography (SWE); hepatectomy; liver failure; indocyanine green (ICG); abdomen

Submitted May 06, 2020. Accepted for publication Dec 03, 2020.

doi: 10.21037/qims-20-640

View this article at: http://dx.doi.org/10.21037/qims-20-640 


\section{Introduction}

Hepatocellular carcinoma (HCC) is one of the most common malignant hepatic tumors worldwide. Since hepatectomy is an effective therapeutic option for a broad range of benign and malignant hepatic tumors, post-hepatectomy liver failure (PHLF) stays on the liver surgeons' agenda. PHLF is one of the most serious complications and major causes of mortality after liver resection. It is correlated with prolonged hospital stay and increased cost of treatment and affects short-term and longterm prognosis (1). Insufficient liver function reserve (LFR) is one of the key factors contributing to PHLF. Therefore, preoperative assessment of LFR is necessary and critical for making appropriate surgery plans and to reduce the risk of PHLF (2). Conventional liver function testing (including bilirubin, albumin, and aspartate aminotransferase) is widely used to assess preoperative hepatic function. While it is a convenient tool, it has relatively low sensitivity and specificity (3). The indocyanine green (ICG) clearance test is another method that has frequently been used as the reference standard for assessing LFR (4). However, it is a blind test without any imaging guidance, and the accuracy of the results can be affected by multiple factors such as perivascular tissue, hemoglobin concentration, and elevated bilirubin levels (5). Also, discrepancies have been reported for the ICG clearance test related to the clinical outcome (6). ICG measurement has high buy-in costs for drug and equipment purchases, and its application is not recommended in patients with thyrotoxicosis or iodine allergy, as ICG contains iodine (7). Furthermore, it has been reported that the value of preoperative ICG clearance testing for predicting PHLF in noncirrhotic patients was not satisfactory (8).

Imaging modalities, such as liver volume measurements by computed tomography (CT), have been used for PHLF prediction in patients scheduled for major hepatectomy. However, the remnant liver volume does not always represent functional reserve, especially in cirrhotic patients $(9,10)$. Gadoxetic acid-enhanced magnetic resonance (MR) imaging can measure LFR directly; however, this approach is expensive and time-consuming $(11,12)$. The nuclear medicine technique ${ }^{99 \mathrm{~m}} \mathrm{Tc}$-galactosyl serum albumin scintigraphy is not routinely performed in most centers and suffers from poor spatial resolution and ionizing radiation exposure (13). Two-dimensional shear wave elastography (2D-SWE), characterized by real-time elastography imaging, non-invasiveness, and reproducible stiffness quantification, was reported to value liver fibrosis assessment significantly $(14,15)$. In theory, the presence of liver fibrosis or cirrhosis results in different degrees of liver injury and LFR impairment. This significant correlation between liver stiffness value (LSV) measured by ultrasound elastography and LFR has been confirmed by some published studies (16). However, few reports on the prediction of PHLF using 2D-SWE imaging in patients undergoing major liver resection $(17,18)$. Furthermore, the performance of 2D-SWE and the ICG clearance test for the assessment of PHLF has not been compared.

This study aimed to investigate and compare the performance of preoperative 2D-SWE and the ICG clearance test for the prediction of PHLF after major liver resection.

\section{Methods}

\section{Patient selection}

The study was approved by the ethics committee of West China Hospital, Sichuan University (No.: 2020755), and was performed following the latest version of the Declaration of Helsinki for Medical Research involving Human Subjects. Informed consent was obtained from all patients. From December 2016 to February 2018, 379 patients who underwent 2D-SWE measurement of background LSV and ICG clearance test before major liver resection were prospectively and consecutively recruited. The exclusion criteria were as follows: (I) patients with a history of liver resection, liver transplantation, or radiofrequency ablation $(\mathrm{n}=49$ ), (II) patients with clinical intervention procedures, for example, transhepatic arterial chemotherapy and embolization (TACE) or chemotherapy before partial hepatectomy ( $\mathrm{n}=148)$, (III) patients with preoperative abnormal serum markers of conventional liver function tests, including abnormal levels of total bilirubin (TB) more than $28.0 \mu \mathrm{mol} / \mathrm{L}, 5$ times higher than normal levels of aspartate transaminase (AST) $35 \mathrm{IU} / \mathrm{L}$, or alanine transaminase (ALT) 40 IU/L $(n=6)$, (IV) time interval greater than 2 months between the ICG clearance test, 2D-SWE examination, and liver resection $(n=4)$. Finally, 172 patients (mean age 54 years, range $25-80$ years, 135 males, 37 females) were enrolled in our study. The median interval time between 2D-SWE examination, ICG clearance test, and liver resection was 4 days (range, 113 days). 


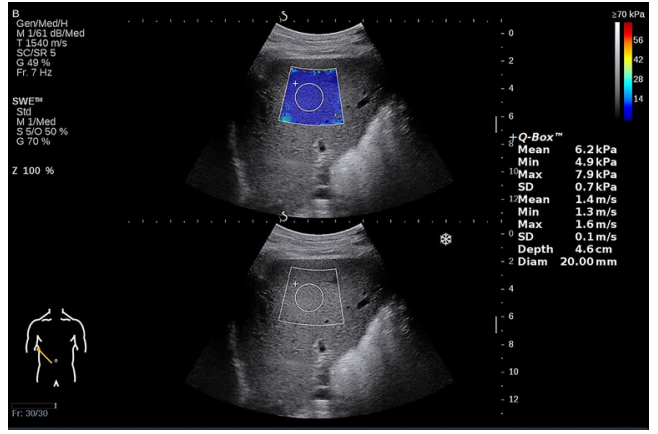

Figure 1 Two-dimensional shear wave elastography (2D-SWE) measurement of liver stiffness value.

\section{D-SWE examination for liver stiffness measurement}

The 2D-SWE examination was performed with an Aixplorer US imaging system (Supersonic Imagine, Axien-Provence) equipped with a broad band convex array transducer (SC6-1). Procedures for 2D-SWE examination were based on the European Federation for Ultrasound in Medicine and Biology (EFSUMB) Guidelines and Recommendations on the Clinical Use of Ultrasound Elastography (19). Patients fasted for at least 6 hours before the 2D-SWE examination. They were placed in a supine position with right arms positioned above the head. The right lobe of liver background parenchyma was observed through the right intercostal or subcostal acoustic window by grayscale ultrasound first. An appropriate probe position was located for identifying the optimal liver parenchymal window. SWE mode was subsequently switched on for elastography, with patients breath-holding in natural breathing cycle for 3-5 seconds. The elasticity imaging box (approximately $4 \mathrm{~cm} \times 3 \mathrm{~cm}$ ) was placed on the liver parenchyma 1-2 cm below the liver capsule, at least $2 \mathrm{~cm}$ away from the liver mass's margin, avoiding visible blood vessels and bile tracts. When the elasticity imaging box was filled with elasticity image signal color stably, a region of interest (ROI) was drawn using a Q-box ${ }^{\mathrm{TM}}$ (diameter, $2 \mathrm{~cm}$ ) and put in the position of the uniform elasticity image signal (Figure 1). At least 5 valid measurements were performed for each patient, and the median elastic modulus $(\mathrm{kPa})$ was recorded. The measurement elasticity imaging box covered by less than half of the elasticity signal or the minimum LSV in the Q-box less than $0.2 \mathrm{kPa}$ was considered invalid. This procedure was performed by 2 ultrasound doctors with more than 3 years of $2 \mathrm{D}$-SWE examination experience. The inter-observer agreement of the 2 doctors for performing
2D-SWE was 0.986 . The intra-observer agreement was 0.990 and 0.991 , respectively.

\section{ICG clearance test}

Patients were placed in a supine position with normal respiration in a quiet environment. ICG solution with a concentration of $5 \mathrm{mg} / \mathrm{mL}$ (sterile water for injection/ICG $=5 \mathrm{~mL}: 25 \mathrm{mg}$ ) was prepared. A standard dose of $0.5 \mathrm{mg} / \mathrm{kg}$ ICG solution was injected within 10 seconds via the median cubital vein. A probe was clamped to the patient's nose, and ICGR15 (ICG retention rate at 15 minutes) was obtained by DDG-3300K (pulse-dye densitometry, PDD).

\section{Definition of PHLF}

PHLF was evaluated according to the International Study Group of Liver Surgery (ISGLS) criteria (20). It defines liver failure by an increased international normalized ratio (INR) and hyperbilirubinemia (according to normal laboratory limits) on or after postoperative day 5 . According to postoperative clinical treatment, the severity of liver failure was classified into 3 levels (A, B, C). No change in the patient's clinical treatment was classified as Grade A. Clinical treatment, which deviated from the routine course but was non-invasive, was classified as Grade B. Grade C required invasive treatment. Postoperative mortality was recorded if a patient died within the postoperative 30 days or died in hospital later than 30 days. The cause of death was recorded.

\section{Statistical analysis}

Statistical tests were conducted using statistical software (SPSS 20.0, Predictive Analytics Software version). The comparison of counting data was conducted by the chisquare test or Fisher's exact probability test. Continuous data were described by the mean and standard deviation or median and interquartile range (IQR). The comparison of continuous data was conducted by analysis of variance (ANOVA) or the Kruskal-Wallis test. The association between variables with PHLF grades was tested using univariate and multivariate logistic regression analysis. Odds ratios were calculated, and $\mathrm{P}<0.1$ was considered significant. The area under the receiver operating characteristic curve (AUC) was also analyzed. AUC is a measure of a test's usefulness in general, where a greater area means a more useful test. Sensitivity and specificity were calculated, and an 
Table 1 Characteristics of the study patients

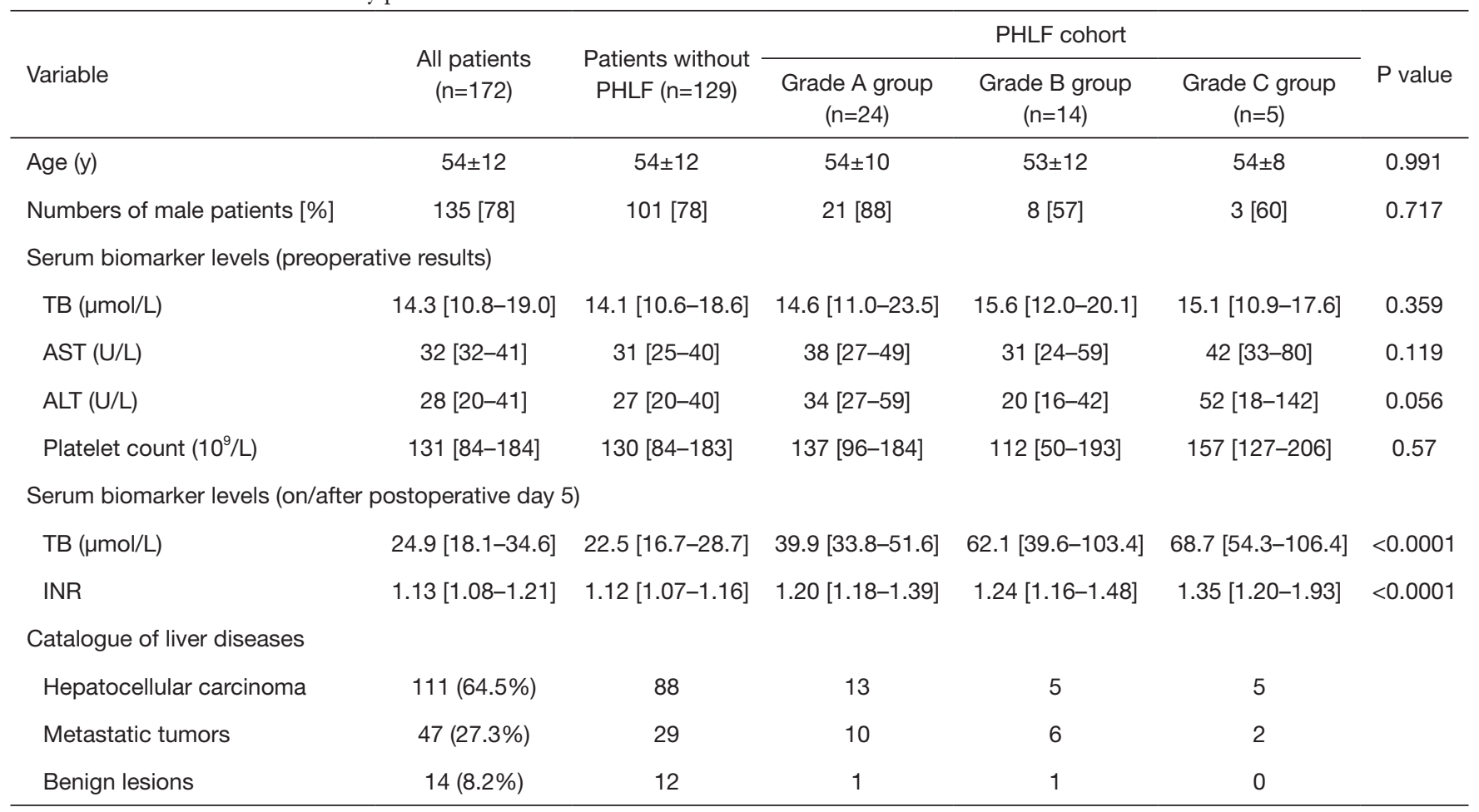

Data were described as means \pm standard deviations or medians [interquartile ranges] as appropriate. Patients were divided into No PHLF, PHLF A, B, C groups according to ISGLS criteria, and statistical analysis was conducted for comparison of these 4 groups. TB, total bilirubin; AST, aspartate transaminase; ALT, alanine aminotransferase; INR, international normalized ratio of prothrombin time; ISGLS, International Study Group of Liver Surgery; PHLF, post-hepatectomy liver failure.

optimal cutoff value that maximized the sum of sensitivity and specificity was determined. A DeLong test was used to compare AUCs. A P value $<0.05$ was considered to indicate a significant result.

\section{Results}

According to the ISGLS criteria, 129 (75\%) patients had normal postoperative INR and TB, and were in the No PHLF group. PHLF occurred in 43 (25.0\%) patients who had an increased INR and elevated TB on or after postoperative day 5 , of which 24 (14.0\%) patients were grade A, 14 (8.1\%) were grade B, and 5 (2.9\%) were grade C. No significant differences were revealed in patient age, gender, and preoperative serum biomarker levels among the No PHLF, PHLF A, PHLF B, and PHLF C groups. A total of $111(64.5 \%)$ patients were pathologically diagnosed with HCC, 47 (27.3\%) were diagnosed with metastatic tumors, and 14 (8.2\%) were diagnosed with benign lesions
(Table 1). Furthermore, 146 (84.9\%) patients had hepatitis B liver background, while $26(15.1 \%)$ had a normal liver background. A total of 132 (76.7\%) patients obtained a pathological diagnosis of liver background. According to Scheuer scores, liver background of these patients was classified as S0 ( $n=17), S 1 \quad(n=4), S 2(n=19), S 3(n=27)$, and S4 ( $\mathrm{n}=65)$. A total of $172(100 \%)$ patients were Child-Pugh $\mathrm{A}$, and their performance status was 0 (normal). No patient death occurred in this study.

\section{LSV of the No PHLF and PHLF A, B, C groups}

Median (IQR) values of LSV in the No PHLF and PHLF A, B, C groups were $9.8 \mathrm{kPa}(7.0-13.8 \mathrm{kPa}), 10.9 \mathrm{kPa}(9.3-$ $12.1 \mathrm{kPa}), 13.0 \mathrm{kPa}(8.4-16.0 \mathrm{kPa})$, and $15.4 \mathrm{kPa}(13.9-$ $22.1 \mathrm{kPa}$ ), respectively. A significant difference in LSV was found among the 4 groups $(\mathrm{P}=0.043)$, and LSV of the PHLF C group was significantly higher than the No PHLF group $(\mathrm{P}=0.025)$ in pairwise comparison (Table 2). 
Table 2 LSV and ICGR15 in study patients

\begin{tabular}{lcccccc}
\hline \multirow{2}{*}{ Variable } & All patients $(n=172)$ & Patients without PHLF & \multicolumn{4}{c}{ PHLF cohort } \\
\cline { 4 - 6 } & & $(n=129)$ & Grade A group $(n=24)$ & Grade B group $(n=14)$ & Grade C group $(n=5)$ \\
\hline ICGR15 (\%) & $4.6[2.6-4.9]$ & $4.1[2.6-6.7]$ & $5.6[4.3-8.2]$ & $6.0[2.9-6.2]$ & $7.0[4.9-12.9]$ & 0.036 \\
LSV $(\mathrm{kPa})$ & $10.5[7.4-14.0]$ & $9.8[7.0-13.8]$ & $10.9[9.3-12.1]$ & $13.0[8.4-16.0]$ & $15.4[13.9-22.1]$ & 0.043 \\
\hline
\end{tabular}

Data were described as medians [interquartile ranges]. Patients were divided into No PHLF, PHLF A, B, C groups according to ISGLS criteria, and statistical analysis was conducted for comparison of these 4 groups. ICGR15, indocyanine green retention rate at 15 minutes; LSV, liver stiffness value; ISGLS, International Study Group of Liver Surgery; PHLF, post-hepatectomy liver failure.

Table 3 Logistic regression analysis for the preoperative risk assessment of PHLF according to ISGLS criteria

\begin{tabular}{lcccc}
\hline \multirow{2}{*}{ Parameter } & Univariate analysis & & \multicolumn{2}{c}{ Multivariate analysis } \\
\cline { 2 - 4 } \cline { 5 - 6 } ICGR15 & Odds ratio & P value & Odds ratio & $0.97(0.93,1.00)$ \\
Liver stiffness & $0.96(0.93,1.00)$ & 0.065 & & 0.084 \\
\hline
\end{tabular}

Data in parentheses are 95\% confidence intervals. $\mathrm{P}<0.1$ was considered as a limit for parameters to enter in the model. ISGLS, International Study Group of Liver Surgery; PHLF, post-hepatectomy liver failure; ICGR15, indocyanine green retention rate at 15 minutes.

\section{ICGR15 of the No PHLF and PHLF A, B, C groups}

Median (IQR) values of ICGR15 in the No PHLF and PHLF A, B, C groups were $4.1 \%(2.6-6.7 \%), 5.6 \%$ $(4.3-8.2 \%), 6.0 \%(2.9-6.2 \%)$, and $7.0 \%(4.9-12.9 \%)$, respectively. A significant difference in ICGR15 was found among the 4 groups $(\mathrm{P}=0.036)$, and ICGR15 of the PHLF $\mathrm{C}$ group was significantly higher than the No PHLF group $(\mathrm{P}=0.001)$ in pairwise comparison (Table 2).

\section{LSV and ICGR15 for assessment of PHLF}

According to the univariate and multivariate logistic regression analyses, LSV and ICGR15 met the limit of significance requirement $(\mathrm{P}<0.1)$, which meant that these 2 parameters were significantly related to PHLF $(\mathrm{P}=0.051$, $\mathrm{P}=0.084$, respectively; Table 3).

For diagnosis of $\geq$ PHLF A using LSV and ICGR15, the AUCs were 0.624 [95\% confidence interval (CI): 0.536$0.712, \mathrm{P}=0.015$ ] and 0.631 (95\% CI: $0.542-0.721, \mathrm{P}=0.01$ ), respectively. No significant difference was revealed between AUCs $(\mathrm{P}=0.17)$. The cutoff values of LSV and ICGR15 were $8.45 \mathrm{kPa}$ (sensitivity $86.1 \%$, specificity $40.3 \%$ ) and $2.95 \%$ (sensitivity $90.7 \%$, specificity $31.0 \%$ ), respectively.

For diagnosis of $\geq$ PHLF B, the AUC of ICGR15 was 0.570 (95\% CI: $0.436-0.704, \mathrm{P}=0.32$ ), which was not significant. The AUC of LSV was 0.699 (95\% CI: $0.576-0.821, \mathrm{P}=0.005)$. The AUC of LSV was significantly higher than that of ICGR15 for the diagnosis of $\geq$ PHLF $\mathrm{B}(\mathrm{P}=0.002)$. The cutoff values of LSV and ICGR15 were $12.70 \mathrm{kPa}$ (sensitivity $68.4 \%$, specificity $71.2 \%$ ) and $5.70 \%$ (sensitivity $52.6 \%$, specificity $63.4 \%$ ), respectively.

For diagnosis of $\geq$ PHLF C, the AUC of ICGR15 was 0.717 (95\% CI: $0.515-0.920, \mathrm{P}=0.098$ ), which was not significant. The AUC of LSV was 0.831 (95\% CI: $0.737-$ $0.925, \mathrm{P}=0.01)$. The AUC of LSV was significantly higher than that of ICGR15 for PHLF C diagnosis ( $\mathrm{P}=0.038)$. The cutoff values of LSV and ICGR15 were $14.90 \mathrm{kPa}$ (sensitivity $80.0 \%$, specificity $80.2 \%$ ) and $6.45 \%$ (sensitivity $80.0 \%$, specificity $71.9 \%$ ), respectively. Details of the data are listed in Table 4, and ROC curves are illustrated in Figure 2.

\section{Discussion}

PHLF remains a major cause of morbidity and mortality after liver resection. Mild-moderate liver failure (grade $\mathrm{A} / \mathrm{B}$; ISGLS grading system) is common following liver resection. PHLF is strongly correlated with prolonged hospital stay and increased medical cost and affects shortterm and long-term prognosis (21). Thus, the prediction of PHLF is of great clinical significance.

LFR is defined as the total of all remnant normal liver cell function after liver injury. In theory, the lower the LFR, the fewer functional liver cells, and the higher the 
Table 4 Liver stiffness value and ICGR15 for preoperative risk assessment of PHLF

\begin{tabular}{|c|c|c|c|c|c|c|}
\hline Parameter & AUC & Cut-off value & Sensitivity (\%) & Specificity (\%) & Likelihood ratio & $P$ value \\
\hline LSV & $0.624(0.536-0.712)$ & $8.45 \mathrm{kPa}$ & $86.1(72.1-94.7)$ & $40.3(31.8-49.3)$ & 1.44 & 0.015 \\
\hline ICGR15 & $0.631(0.542-0.721)$ & $2.95 \%$ & 90.7 (77.9-97.4) & $31.0(23.2-39.8)$ & 1.31 & 0.01 \\
\hline \multicolumn{7}{|c|}{$\geq$ PHLF B $(n=153$ vs. $n=19)$} \\
\hline ICGR15 & $0.570(0.436-0.704)$ & $5.70 \%$ & $52.6(28.9-75.6)$ & $63.4(55.2-71.0)$ & 1.44 & 0.32 \\
\hline \multicolumn{7}{|c|}{$\geq$ PHLF C ( $n=167$ vs. $n=5)$} \\
\hline LSV & $0.831(0.737-0.925)$ & $14.90 \mathrm{kPa}$ & $80.0(28.4-99.5)$ & $80.2(73.4-86.0)$ & 4.05 & 0.01 \\
\hline ICGR15 & $0.717(0.515-0.920)$ & $6.45 \%$ & $80.0(28.4-99.5)$ & $71.9(64.4-78.9)$ & 2.84 & 0.098 \\
\hline
\end{tabular}

Data in parentheses are 95\% confidence intervals. PHLF, post-hepatectomy liver failure; ICGR15, indocyanine green retention rate at 15 minutes; LSV, liver stiffness value; AUC, area under the receiver operating characteristic curve.

A

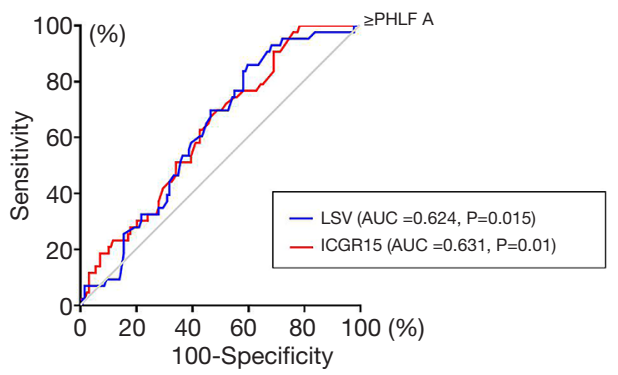

C

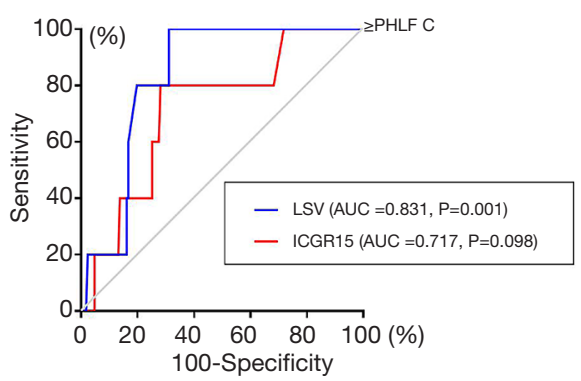

B

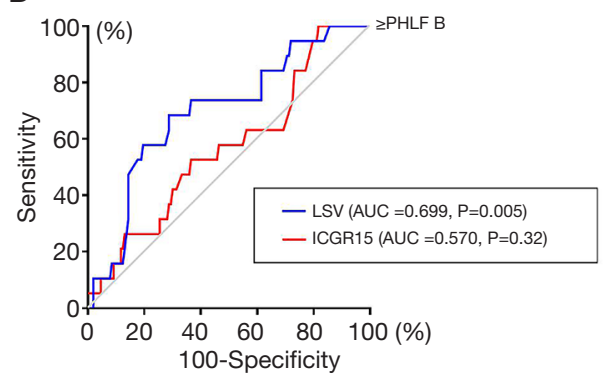

Figure 2 Comparison of diagnostic efficacy between liver stiffness value (LSV) and indocyanine green retention rate at 15 minutes (ICGR15) for the prediction of $(\mathrm{A}) \geq$ post-hepatectomy liver failure (PHLF) grade A, (B) $\geq$ PHLF grade $\mathrm{B},(\mathrm{C}) \geq$ PHLF grade C.

risk of developing PHLF. Accordingly, the risk of PHLF can be indirectly reflected by the LFR assessment. The ICG clearance test is the most acknowledged approach for evaluating LFR. ICG is a water-soluble, FDA approved dye, which can be selectively taken up by liver cells and eliminated in its original form through bile without any metabolic change or extrahepatic intake. Due to this exclusive hepatic clearance, the ICG retention or elimination rate is a widely used marker for LFR evaluation $(5,22)$. In this study, preoperative ICGR15 elevated with the severity of PHLF. ICGR15 of the PHLF C group was significantly higher than that of the No PHLF group. 
This implies a close association between ICGR15 and the severity of PHLF. The AUCs of ICGR15 for diagnosing $\geq$ PHLF A, $\geq$ PHLF $B$, and $\geq$ PHLF C in this study were 0.631 $(\mathrm{P}=0.01), 0.570(\mathrm{P}=0.32)$, and $0.717(\mathrm{P}=0.098)$ respectively. These results indicated that the efficacy of preoperative ICGR15 for diagnosing $\geq$ PHLF A was slightly better than that for diagnosing $\geq$ PHLF B and $\geq$ PHLF C. However, in general, the diagnostic performance of ICGR15 was not good. This resulted from Cem et al., who demonstrated that the efficacy of the ICG clearance test for PHLF prediction in noncirrhotic patients was unsatisfactory $(\mathrm{AUC}=0.51$, $\mathrm{P}=0.90$ ) (8). Another study by Wong et al. also supported the finding that ICGR15 failed to predict the occurrence of major complications after liver resection (23). The possible reason for the ICG clearance test's low efficacy to predict PHLF might be the principle of the ICG clearance test. A preoperative ICG clearance test reflects the LFR of the whole liver rather than a certain liver lobe. After partial liver hepatectomy, preoperative whole liver LFR may not represent remnant liver lobe LFR due to heterogeneous liver background. Furthermore, the testing principle of PDD (for ICGR15 measurement) is based on twowavelength near-infrared light, and the slight shake of a tested body part such as the nose or finger will lead to an inaccurate ICGR15 result (24).

SWE is a non-invasive, convenient, and repeatable imaging modality for liver fibrosis assessment. The high efficacy of SWE for diagnosing $\geq \mathrm{F} 2$ liver fibrosis has been confirmed over the past decade and has been recommended for clinical application $(15,19)$. In theory, different severity of fibrosis leads to different degrees of LFR impairment. Recent research has revealed that SWE's liver stiffness was closely correlated with the ICG clearance test index and Child-Pugh score $(16,25)$. Therefore, SWE may have the potential to reflect LFR and predict PHLF indirectly. Our present study investigated the performance of preoperative 2D-SWE for PHLF prediction and whether it is superior to the ICG clearance test. The study results showed that LSV increased when PHLF was worse. The LSV of the PHLF C group was significantly higher than that of the No PHLF group (9.8 vs. $15.4 \mathrm{kPa}, \mathrm{P}=0.025)$, indicating that patients with more severe liver fibrosis were more likely to develop serious PHLF after liver resection. The AUCs of preoperative 2D-SWE for diagnosing $\geq$ PHLF A, $\geq$ PHLF B, and $\geq$ PHLF C were $0.624(\mathrm{P}=0.015), 0.699(\mathrm{P}=0.005)$, and 0.831 $(\mathrm{P}=0.01)$, respectively, which indicated that this test could significantly predict PHLF. 2D-SWE showed comparable efficacy for $\geq$ PHLF A prediction and better efficacy for $\geq$ PHLF B and $\geq$ PHLF C prediction compared to the ICG clearance test. A study conducted by Shen et al. showed similar results regarding 2D-SWE for predicting $\geq$ PHLF A and $\geq$ PHLF B, as AUCs were 0.72 and 0.76 respectively (18), but PHLF C was not mentioned in their study. Another study found that the AUC of 2D-SWE for outcome prediction in patients with acute-on-chronic hepatitis B liver failure was 0.72 (26). Nishio et al. used Virtual Touch Tissue Quantification to predict $\geq$ PHLF A, $\geq$ PHLF B, and $\geq$ PHLF C, and the AUCs were $0.67,0.78$, and 0.74 , respectively (27). Lei et al. reported a much higher AUC (0.86) of transient elastography (TE, Fibroscan) for predicting PHLF in patients with hepatitis B-related HCC (17). However, the definition of PHLF in their study was different from that defined by the ISGLS, and they did not perform PHLF risk classification. In the current study, the 2D-SWE cut-off value of $8.45 \mathrm{kPa}$ predicted $\geq$ PHLF A with high sensitivity (sensitivity $86.1 \%$, specificity $40.3 \%$ ). The cut-off value of $12.70 \mathrm{kPa}$ predicted $\geq$ PHLF B with increased specificity (sensitivity $68.4 \%$, specificity $71.2 \%$ ), and the cut-off value of $14.90 \mathrm{kPa}$ predicted $\geq$ PHLF C with both good sensitivity and specificity (sensitivity $80.0 \%$, specificity $80.2 \%$ ), indicating that for patients with preoperative LSV (measured by 2D-SWE) higher than $14.9 \mathrm{kPa}$, their surgery plan might be reconsidered to avoid PHLF C. Other study results also suggested that a higher LSV was a good and reliable preoperative risk predictor for PHLF $(17,18,27,28)$. SWE demonstrated good performance for PHLF prediction, but it was noted that the reported AUC and cut-off value of SWE for PHLF prediction varied among different studies. This may be due to different ultrasound elastography equipment being used, an inconsistent definition of PHLF, and mixed liver etiology.

Limitations exist in our study. The number of PHLF C cases was relatively small, and a greater number of these cases should be included in future studies. Furthermore, it is generally accepted that factors such as intraoperative blood loss, operative time, and residual liver volume can influence PHLF outcome. However, in a real clinical setting, these factors are not known before surgery; therefore, they were not included in this study, which was for preoperative PHLF prediction. This study retrospectively investigated and compared the efficacy of 2D-SWE and the ICG clearance test for preoperative PHLF prediction in the same cohort of patients undergoing major liver resection. Therefore, a prospective, larger-population study should be 
performed to validate the present results.

In conclusion, 2D-SWE demonstrates the potential to aid in the prediction of the severity of PHLF. Our findings suggest that the performance of 2D-SWE is better than the ICG clearance test.

\section{Acknowledgments}

We thank Dr. Jibin Liu for professional advice in the preparation of the article.

Funding: This study is supported by National Natural Science Foundation of China (NSFC) (Grant No. 82001833), Post-Doctor Research Project, West China Hospital, Sichuan University (Grant No. 2019HXBH014), National Natural Science Foundation of China (NSFC) (Grant No. 82071940), Sichuan Science and Technology Program (Grant No. 2020YFS0211).

\section{Footnote}

Conflicts of Interest: All authors have completed the ICMJE uniform disclosure form (available at http://dx.doi. org/10.21037/qims-20-640). The authors have no conflicts of interest to declare.

Ethical Statement: The study was approved by the ethics committee of West China Hospital, Sichuan University (No.: 2020755), and was performed following the latest version of the Declaration of Helsinki for Medical Research involving Human Subjects. Informed consent was obtained from all patients.

Open Access Statement: This is an Open Access article distributed in accordance with the Creative Commons Attribution-NonCommercial-NoDerivs 4.0 International License (CC BY-NC-ND 4.0), which permits the noncommercial replication and distribution of the article with the strict proviso that no changes or edits are made and the original work is properly cited (including links to both the formal publication through the relevant DOI and the license). See: https://creativecommons.org/licenses/by-nc-nd/4.0/.

\section{References}

1. Lock JF, Reinhold T, Malinowski M, Pratschke J, Neuhaus P, Stockmann M. The costs of postoperative liver failure and the economic impact of liver function capacity after extended liver resection: a single-center experience.
Langenbecks Arch Surg 2009;394:1047-56.

2. Cieslak KP, Runge JH, Heger M, Stoker J, Bennink RJ, van Gulik TM. New Perspectives in the Assessment of Future Remnant Liver. Dig Surg 2014;31:255-68.

3. Zou H, Yang X, Li QL, Zhou QX, Xiong L, Wen Y. A Comparative Study of Albumin-Bilirubin Score with Child-Pugh Score, Model for End-Stage Liver Disease Score and Indocyanine Green R15 in Predicting Posthepatectomy liver failure for Hepatocellular Carcinoma Patients. Dig Dis 2018;36:236-43.

4. Hoekstra LT, de Graaf W, Nibourg GAA, Heger M, Bennink RJ, Stieger B, van Gulik TM. Physiological and biochemical basis of clinical liver function tests: a review. Ann Surg 2013;257:27-36.

5. Ott P. Hepatic elimination of indocyanine green with special reference to distribution kinetics and the influence of plasma protein binding. Pharmacol Toxicol 1998;83 Suppl 2:1-48.

6. Rassam F, Olthof PB, Bennink RJ, van Gulik TM. Current Modalities for the Assessment of Future Remnant Liver Function. Visc Med 2017;33:442-8.

7. Levesque E, Martin E, Dudau D, Lim C, Dhonneur G, Azoulay D. Current use and perspective of indocyanine green clearance in liver diseases Anaesth Crit Care Pain Med 2016;35:49-57.

8. Ibis C, Albayrak D, Sahiner T, Soytas Y, Gurtekin B, Sivrikoz N. Value of Preoperative Indocyanine Green Clearance Test for Predicting Post-Hepatectomy Liver Failure in Noncirrhotic Patients. Med Sci Monit 2017;23:4973-80.

9. Lim MC, Tan CH, Cai J, Zheng J, Kow AW. CT volumetry of the liver: Where does it stand in clinical practice? Clin Radiol 2014;69:887-95.

10. Witowski J, Wake N, Grochowska A, Sun Z, Budzyński A, Major P, Popiela TJ, Pędziwiatr M. Investigating accuracy of 3D printed liver models with computed tomography. Quant Imaging Med Surg 2019;9:43-52.

11. Nakagawa M, Namimoto T, Shimizu K, Morita K, Sakamoto F, Oda S, Nakaura T, Utsunomiya D, Shiraishi $\mathrm{S}$, Yamashita Y. Measuring hepatic functional reserve using T1 mapping of Gd-EOB-DTPA enhanced 3T MR imaging: A preliminary study comparing with 99mTc GSA scintigraphy and signal intensity based parameters. Eur J Radiol 2017;92:116-23.

12. Verde F, Romeo V, Maurea S. Advanced liver imaging using MR to predict outcomes in chronic liver disease: a shift from morphology to function liver assessment. Quant Imaging Med Surg 2020;10:805-7. 
13. Mizutani Y, Hirai T, Nagamachi S, Nanashima A, Yano K, Kondo K, Hiyoshi M, Imamura N, Terada T. Prediction of Post-hepatectomy liver failure Proposed by the International Study Group of Liver Surgery: Residual Liver Function Estimation With 99mTc-Galactosyl Human Serum Albumin Scintigraphy. Clin Nucl Med 2018;43:77-81.

14. Samir AE, Dhyani M, Vij A, Bhan AK, Halpern EF, Méndez-Navarro J, Corey KE, Chung RT. Shear-Wave Elastography for the Estimation of Liver Fibrosis in Chronic Liver Disease: Determining Accuracy and Ideal Site for Measurement. Radiology 2015;274:888-96.

15. Gao Y, Zheng J, Liang P, Tong M, Wang J, Wu C, He X, Liu C, Zhang S, Huang L, Jiang T, Cheng C, Meng F, Mu X, Lu Y, Li Y, Ai H, Qiao X, Xie XY, Wang W, Yin LP, Wu YY, Zheng R. Liver Fibrosis with Two-dimensional US Shear-Wave Elastography in Participants with Chronic Hepatitis B: A Prospective Multicenter Study. Radiology 2018;289:407-15.

16. Sun XL, Liang LW, Cao H, Men Q, Hou KZ, Chen Z, Zhao YE. Liver reserve function assessment by acoustic radiation force impulse imaging. World J Gastroenterol 2015;21:9648-9655.

17. Lei JW, Ji XY, Hong JF, Li WB, Chen Y, Pan Y, Guo J. Prediction of post-hepatectomy liver failure using transient elastography in patients with hepatitis B related hepatocellular carcinoma. BMC Gastroenterol 2017;17:171.

18. Shen Y, Zhou C, Zhu G, Shi G, Zhu X, Huang C, Zhou J, Fan J, Ding H, Ren N, Sun HC. Liver Stiffness Assessed by Shear Wave Elastography Predicts Postoperative Liver Failure in Patients with Hepatocellular Carcinoma. J Gastrointest Surg 2017;21:1471-9.

19. Cosgrove D, Piscaglia F, Bamber J, Bojunga J, Correas JM, Gilja OH, Klauser AS, Sporea I, Calliada F, Cantisani V, D’Onofrio M, Drakonaki EE, Fink M, FriedrichRust M, Fromageau J, Havre RF, Jenssen C, Ohlinger R, Săftoiu A, Schaefer F, Dietrich CF; EFSUMB. EFSUMB guidelines and recommendations on the clinical use of ultrasound elastography. Part 2: Clinical applications. Ultraschall Med 2013;34:238-53.

20. Rahbari NN, Garden OJ, Padbury R, Brooke-Smith M, Crawford M, Adam R, Koch M, Makuuchi M, Dematteo RP, Christophi C, Banting S, Usatoff V, Nagino M, Maddern G, Hugh TJ, Vauthey JN, Greig P, Rees M, Yokoyama Y, Fan ST, Nimura Y, Figueras J, Capussotti L, Büchler MW, Weitz J. Post-hepatectomy liver failure: a definition and grading by the International Study Group of Liver Surgery (ISGLS). Surgery 2011;149:713-24.

21. Gilg S, Sandström P, Rizell M, Lindell G, Ardnor B, Strömberg C, Isaksson B. The impact of post-hepatectomy liver failure on mortality: a population-based study. Scand J Gastroenterol 2018;53:1335-9.

22. Heimbach JK, Kulik LM, Finn RS, Sirlin CB, Abecassis MM, Roberts LR, Zhu AX, Murad MH, Marrero JA. AASLD guidelines for the treatment of hepatocellular carcinoma. Hepatology 2018;67:358-80.

23. Wong JS, Wong GL, Chan AW, Wong VW, Cheung YS, Chong CN, Wong J, Lee KF, Chan HL, Lai PB. Liver Stiffness Measurement by Transient Elastography as a Predictor on Posthepatectomy Outcomes. Ann Surg 2013;257:922-8.

24. Imai T, Takahashi K, Goto F, Morishita Y. Measurement of blood concentration of indocyanine green by pulse dye densitometry - Comparison with the conventional spectrophotometric method. J Clin Monit Comput 1998;14:477-84.

25. Wang J, Wang Q, Yu G, She Q, Zhang W, Zhang J. Correlation Between Liver Stiffness Measured by ShearWave Elastography and Child-Pugh Classification. J Ultrasound Med 2018;37:2191-9.

26. Jin JY, Zheng YB, Zheng J, Liu J, Mao YJ, Chen SG, Gao ZL, Zheng RQ. 2D shear wave elastography combined with MELD improved prognostic accuracy in patients with acute-on-chronic hepatitis B liver failure. Eur Radiol 2018;28:4465-74.

27. Nishio T, Taura K, Koyama Y, Tanabe K, Yamamoto G, Okuda Y, Ikeno Y, Seo S, Yasuchika K, Hatano E, Okajima H, Kaido T, Tanaka S, Uemoto S. Prediction of post-hepatectomy liver failure based on liver stiffness measurement in patients with hepatocellular carcinoma. Surgery 2016;159:399-408.

28. Han H, Hu H, Xu YD, Wang WP, Ding H, Lu Q. Liver failure after hepatectomy: A risk assessment using the prehepatectomy shear wave elastography technique. Eur J Radiol 2017;86:234-40.

Cite this article as: Qiu T, Fu R, Ling W, Li J, Song J, Wu Z, Shi Y, Zhou Y, Luo Y. Comparison between preoperative two-dimensional shear wave elastography and indocyanine green clearance test for prediction of post-hepatectomy liver failure. Quant Imaging Med Surg 2021;11(5):1692-1700. doi: 10.21037/qims-20-640 